\title{
DIAGNOSIS OF A POORLY PERFORMING LIQUID HYDROGEN BULK STORAGE SPHERE
}

\author{
A. Krenn \\ Fluids Division, Cryogenics Branch, NE-F7 \\ NASA Kennedy Space Center, Florida 32899 U.S.A
}

\begin{abstract}
There are two 850,000 gallon Liquid Hydrogen (LH2) storage spheres used to support the Space Shuttle Program; one residing at Launch Pad A and the other at Launch Pad B. The LH2 Sphere at Pad B has had a high boiloff rate since being brought into service in the 1960's. The daily commodity loss was estimated to be approximately double that of the Pad A sphere, and well above the minimum required by the sphere's specification. Additionally, after being re-painted in the late 1990's a "cold spot" appeared on the outer sphere which resulted in a poor paint bond, and mold formation. Thermography was used to characterize the area, and the boiloff rate was continually evaluated. All evidence suggested that the high boiloff rate was caused by an excessive heat leak into the inner sphere due to an insulation void in the annulus. Pad B was recently taken out of Space Shuttle program service which provided a unique opportunity to diagnose the sphere's poor performance. The sphere was drained and inerted, and then opened from the annular relief device on the top where a series of boroscoping operations were accomplished. Boroscoping revealed a large Perlite insulation void in the region of the sphere where the cold spot was apparent. Perlite was then trucked in and offloaded into the annular void region until the annulus was full. The sphere has not yet been brought back into service.
\end{abstract}

KEYWORDS: cryogenic spheres, thermal insulation, perlite 


\section{A BRIEF DESCRIPTION OF THE SUBJECT CRYOGENIC STORAGE SPHERE}

In the mid 1960s, Chicago Bridge and Iron built an 850,000 gallon Liquid Hydrogen Storage Sphere on each of the two Launch Complex 39 Pads (A \& B) at Kennedy Space Center in support of the Apollo Program. The two spheres continued to operate under the Space Shuttle Program. A 61.5 foot diameter stainless steel inner sphere is concentrically set inside a 70 foot diameter carbon steel outer sphere. The resulting 58,000 cubic foot annular space is filled with perlite insulation with a compacted density of $8-9.5 \mathrm{lb} / \mathrm{cu} . \mathrm{ft}$ and is held under a cold vacuum of $10-15$ microns. Losses are not to exceed $0.075 \%$ of capacity (637.5 gallons/day) according to the specification. In order to estimate boiloff rate, several months of quiescence is required, because the liquid level indication on an active sphere is volatile. Actual losses were estimated for both spheres using historical data and revealed that the Pad A boiloff rate has consistently been around 300 gallons/day regardless of the sphere's fill level. On the other hand, the Pad B boiloff rate varied with fill level but averaged nearly 700 gallons/day in the mid 1970 s, and by 2008 was up to 1000 gallons/day. The Pad B data shows that there was no significant increase in the boiloff rate from 2001 to 2009 , but frequent sphere activity and varying fill levels resulted in insufficient historical data to determine if the noted increase took place slowly over time, or occurred all at once.

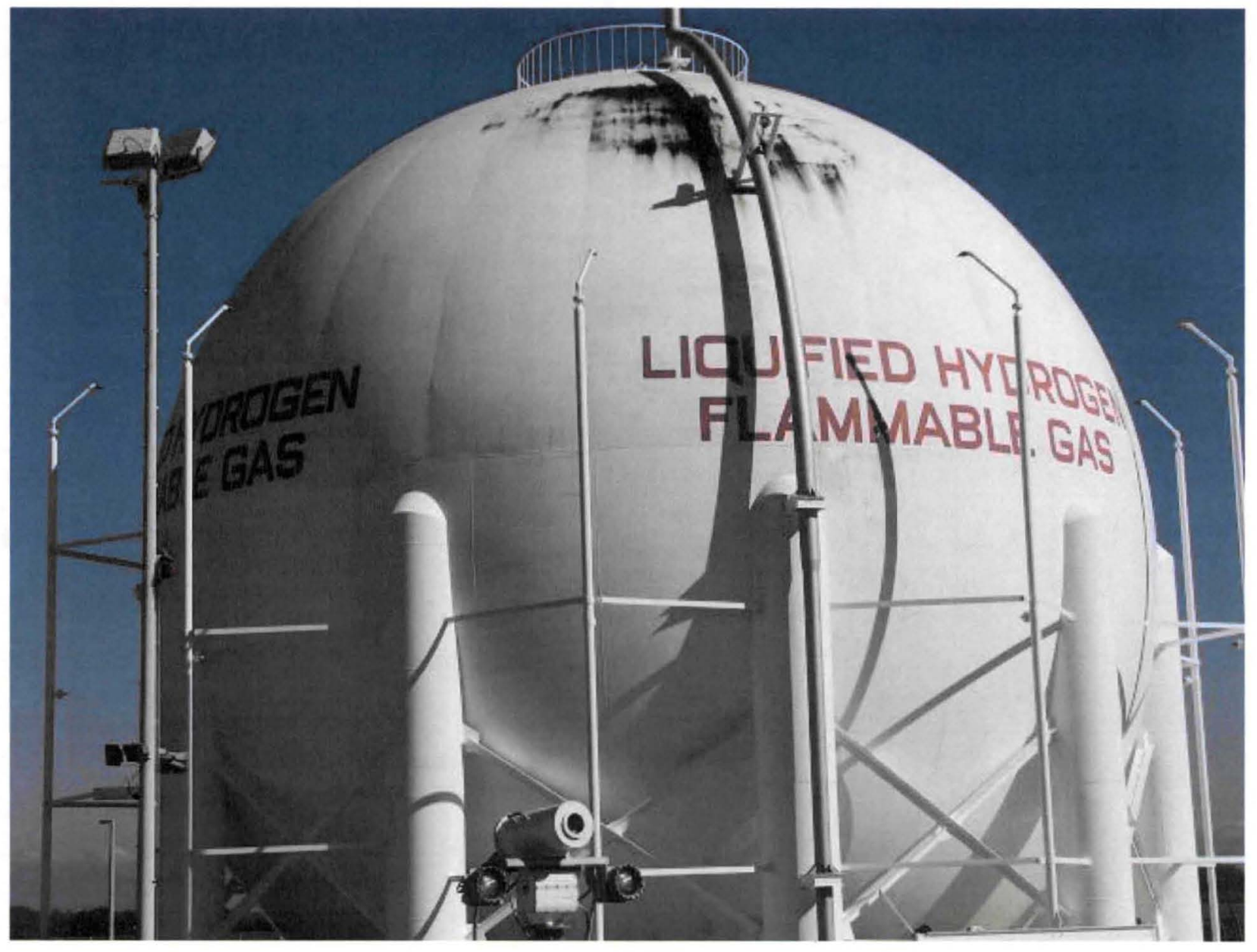

FIGURE 1. LC-39B Liquid Hydrogen Storage Sphere with Mold Spot 


\section{ABNORMAL OBSERVATIONS}

In addition to the out of spec boiloff rate, several other observations were made which provided evidence that a problem existed, and that further investigation would be required. At some point in the late 1990's, a mold spot appeared on the external surface of the outer jacket (see figure 1). The area with mold was straddled by the vent line, and quickly re-appeared when washed away which indicated that this region of the sphere remained consistently below the dewpoint temperature. Additionally, difficulties were encountered during storage sphere re-painting operations. During primer application, contractor personnel reported insufficient adhesion in a localized area due to an inability to maintain a moisture free surface. The surface of the sphere was reported to be cool to the touch causing condensation to constantly form over the area. The area of primer adhesion difficulty coincided with the area which was moldy prior to paint removal.

Hurricanes are an occasional reality in Florida, and as such, Kennedy Space Center has established hurricane procedures. For the Liquid Hydrogen Systems, the normally vented spheres are "locked-up" prior to personnel evacuation in order to prevent moisture and/or air intrusion. The storage spheres remain in a non vented configuration until personnel are cleared to return to the area. Typically, there is no cause for concern during this process, but in August of 2006, Hurricane Ernesto triggered hurricane securing and a subsequent evacuation which revealed an unforeseen vulnerability: Sphere pressure rose at an extremely high rate. The LH2 Sphere at Pad B was filled to 820,000 gallons at the time of its lock-up. Ernesto's impacts were minimal, and personnel were back at work after only one day of evacuation. The sphere was vented after being locked up for a mere 30 hours, but the pressure had risen from 1 psig to 47 psig. Modeling suggests that if the lockup period had lasted 48 hours or longer, the storage sphere would have exceeded 90 psig causing the storage sphere relief valve to lift. This pressure rise was much higher than would be expected in a nominal sphere due to the high heat leak near the top of the sphere.

An extensive investigation into the possible causes of these abnormal conditions was prevented by the operational status of the storage sphere, however; advances in thermographic imaging allowed for some additional data collection. Several sets of thermal images were taken from many angles in different ambient conditions over several years. When these images were analyzed, they suggested that the anomalous area was approximately 5 - 8 degrees $\mathrm{F}$ colder than the rest of the external shell (see figure 2).

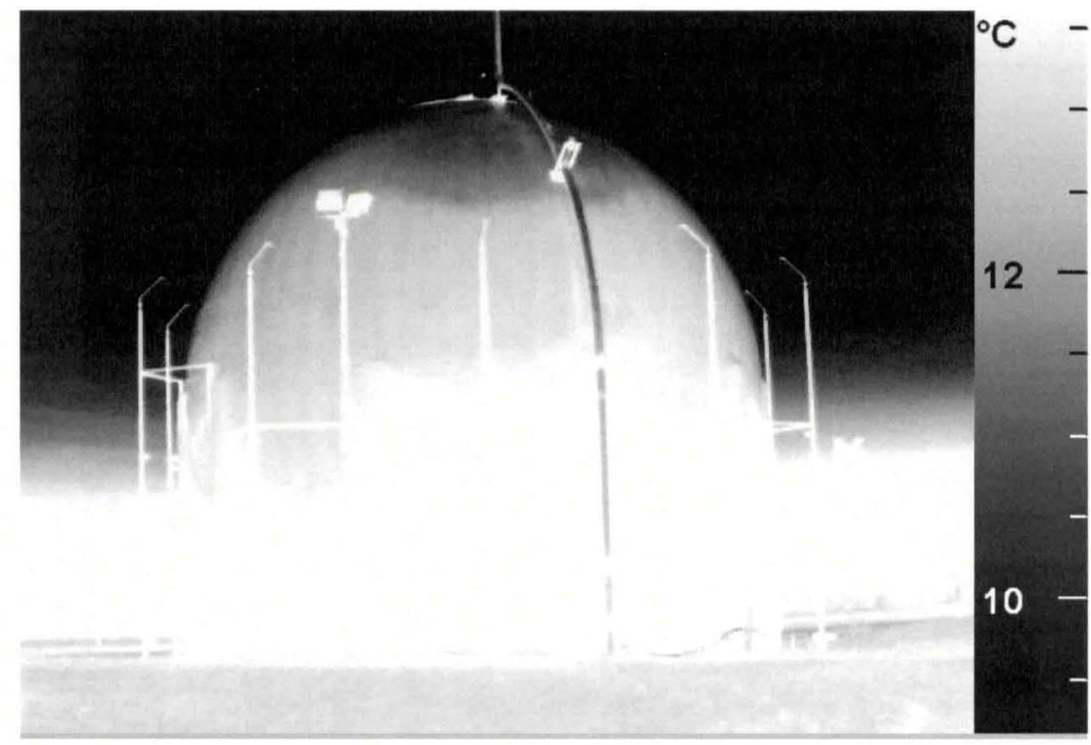

FIGURE 2. Thermal Image of Anomalous Area 


\section{INVESTIGATION AFTER SPHERE DECOMMISSIONING}

The LH2 Sphere at Pad B was decommissioned and turned over to the Constellation Program in 2009. Later that year, the decision was made to drain and inert the sphere to support refurbishment efforts. Sphere empting was completed in January of 2010 followed immediately by inerting in February and release of annular vacuum in March. By April, the sphere had warmed to ambient temperature and was ready to be investigated.

The 12 inch annular relief device was opened up, and the condition of the perlite was observed and photographed. It was approximately 17.5 inches below the top of the outer sphere and had a downward slope in the direction of the vent piping (see figure 3 ). Samples of the perlite were then taken. A sample was also taken from the bottom of the sphere by cutting off the 2 inch drain port cap. Perlite flowed freely into a collection container, then a fiberglass plug was installed, and the cap was welded back into place. Both samples were white and fluffy, and subsequent analysis identified no contaminants. A compaction test of the samples validated that the existing perlite compacted density is in the original specification range of $8-9.5 \mathrm{lb} / \mathrm{cu}$. ft.

After perlite samples were gathered from the top of the sphere via the 12 inch annular relief opening, a boroscope was inserted in order to ascertain the cause of the high heat leak (see figure 4). Several difficulties were encountered during the boroscoping process. When the lens contacted perlite, a "white out" occurred, and the boroscope had to be removed and cleaned before visibility could be restored. The curvature of the sphere, and the fluffiness of the perlite made white outs a frequent challenge. To combat this challenge, the boroscope was run through a curved tube and a Teflon "ski" was used to keep the boroscope head above the perlite. In addition to perlite, there are pipes, supports, pumpdown spirature, and other structures inside the annular region. All of these structures served as interferences to the boroscope and limited its travel. After several attempts on multiple days, using various lighting techniques, the boroscope recorded conclusive evidence of the cause of the sphere's high heat leak. As suspected, there was a huge void in the insulation. It was difficult to detect at first because perlite was mounded up on the top of the sphere, but once the boroscope was able to successfully travel some distance, the void was apparent. Due to the difficulties boroscoping, the exact volume of the void was undeterminable, but was estimated to be between 7,000 - 14,000 cubic feet (12-24\%).

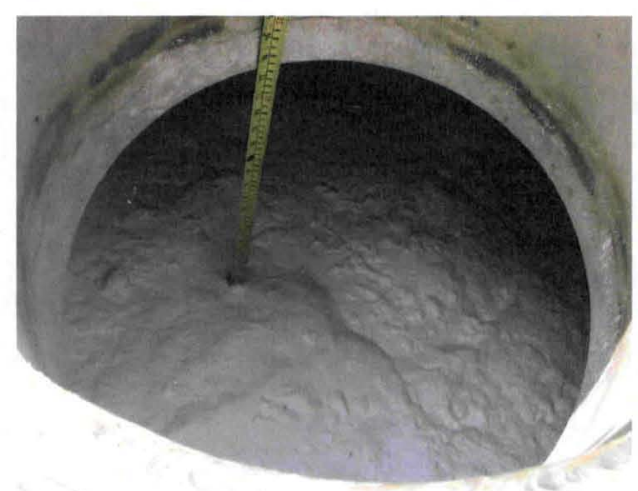

FIGURE 3. First Look Inside Annulus

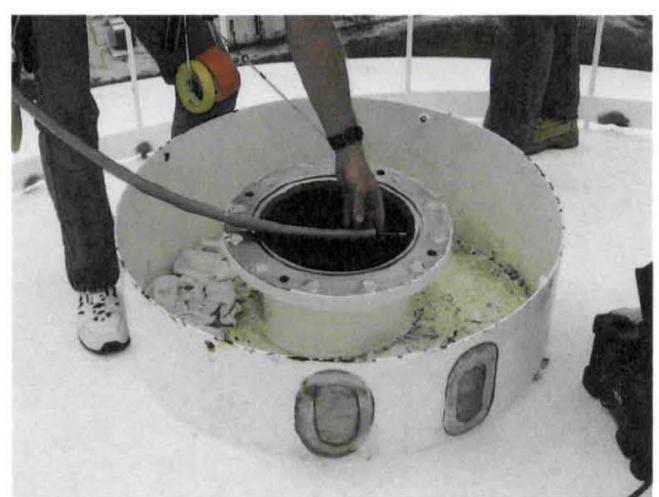

FIGURE 4. Boroscope Being Inserted Into the Annular Relief Opening 


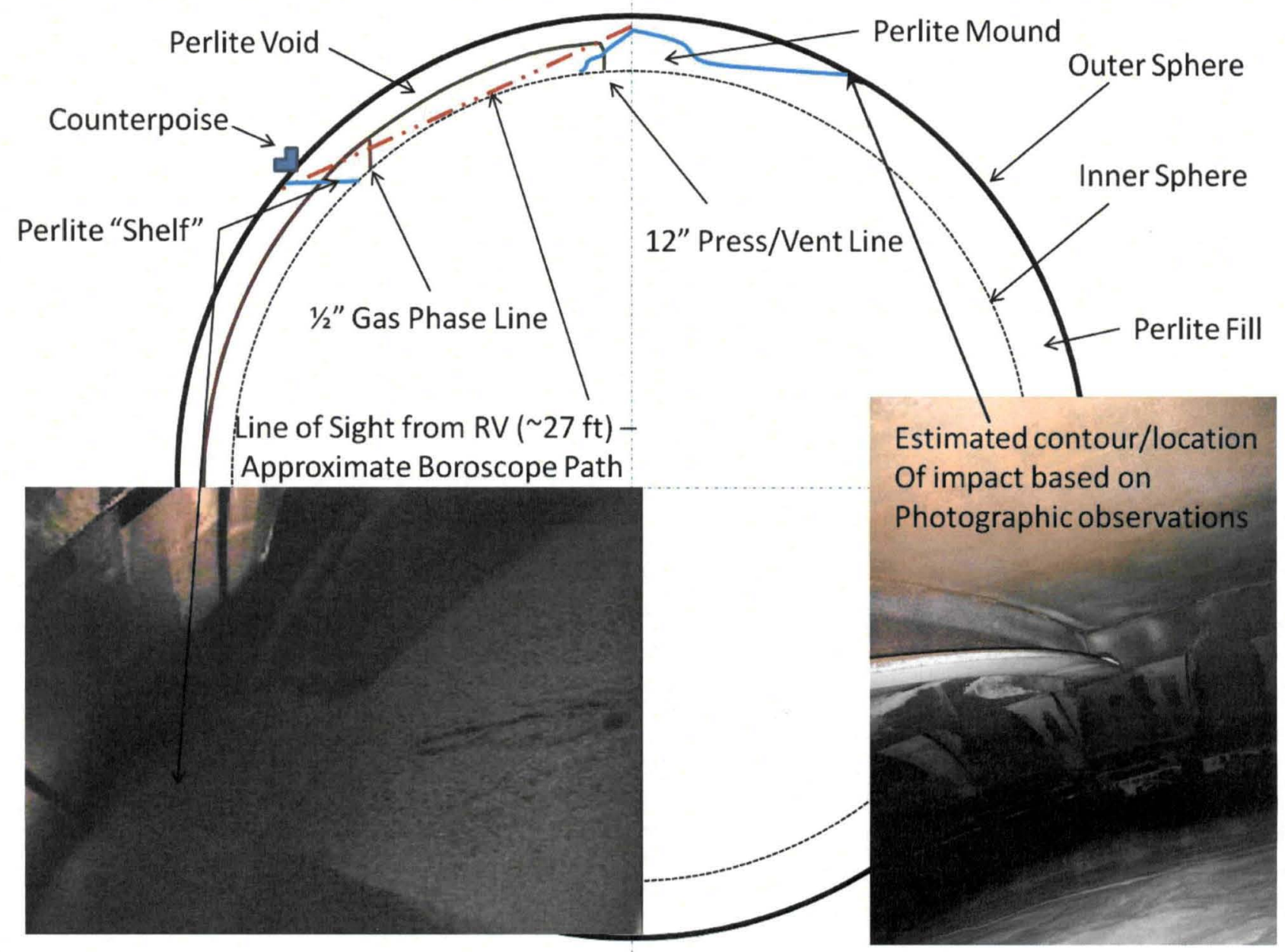

FIGURE 5. Estimated Contour of Perlite Based on Boroscope Results

\section{PERLITE TOP OFF PROCESS}

With verification of the pristine condition of the existing perlite, and video evidence of a void, the decision was made to fill the void with new perlite. The possibility of using an alternative advanced insulation was considered, but ultimately rejected. Glass beads have been shown to result in a marked reduction in boiloff, but the area of concern in this sphere was relatively small. As a result, the use of glass beads instead of perlite was only estimated to reduce boiloff by an additional 2\%. Additionally, there was some concern over potential "internal erosion" due to differences in particle size and shape. Glass bead particles are smaller than perlite particles, and vibration testing would have been required to ensure the glass beads would not settle down through the perlite resulting in potential compaction near the bottom of the sphere.

When the sphere was originally filled with perlite after fabrication in the $1960 \mathrm{~s}$, a vacuum was pulled on the annulus and the perlite was cracked on-site. In 2010, less than 15,000 cubic feet of perlite was required which rendered the vacuum/on-site cracking method cost prohibitive. As a result, perlite was cracked off-site, trucked in and blown into the annulus by an air compressor. Perlite with a compacted density of $10.5-12.5 \mathrm{lb} / \mathrm{cu}$. ft was purchased with the intention of ending up with a functional density comparable to that which was already in the sphere. The theory being, perlite installed under vacuum would compact more than blown perlite. Air samples were taken from the compressor each day prior to use, and the moisture content was consistently less than 200 PPM. Perlite samples 
were taken from each truck prior to use and post installation tests confirmed no hydrocarbons or significant contaminants were present. A vibration device was used on the outer shell to try and settle the blown perlite into any hard to reach void areas.

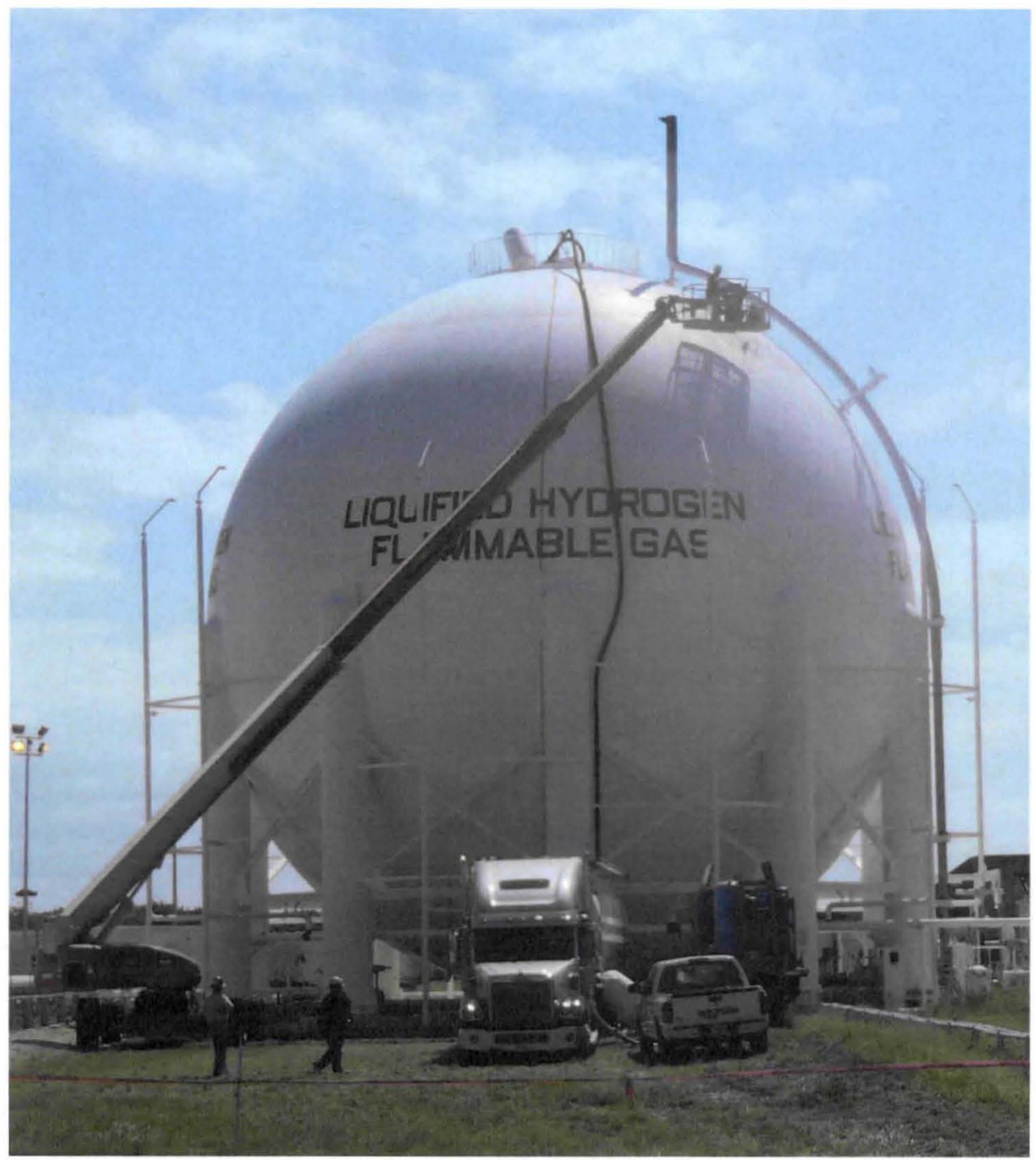

FIGURE 6. Perlite Top-off Set-up

The perlite contractor's typical fill verification method is to provide a person at the fill location to notify the ground crew when perlite begins to fill the filter sock (which is kept around the fill location to prevent perlite from escaping). Due to the history of the LC-39B sphere, there was a strong desire for a more affirmative method of verification. In the 1960 s, the perlite was installed with a minimum temperature of 200 degrees F. This was to ensure no moisture absorption, and was relatively easy to maintain due to the perlite being cracked on-site. Initially, the plan was to use thermography, which had helped identify the problem to begin with, to verify the lower extent of the void was reached. However, blowing perlite in from trucks resulted in near ambient temperature perlite going into the sphere and rendered any use of thermography ineffective. A combination of limited boroscope viewing coupled with still photography taken from a tethered camera inserted into both the fill and vent openings was used to establish that the annular region was as full as possible. 


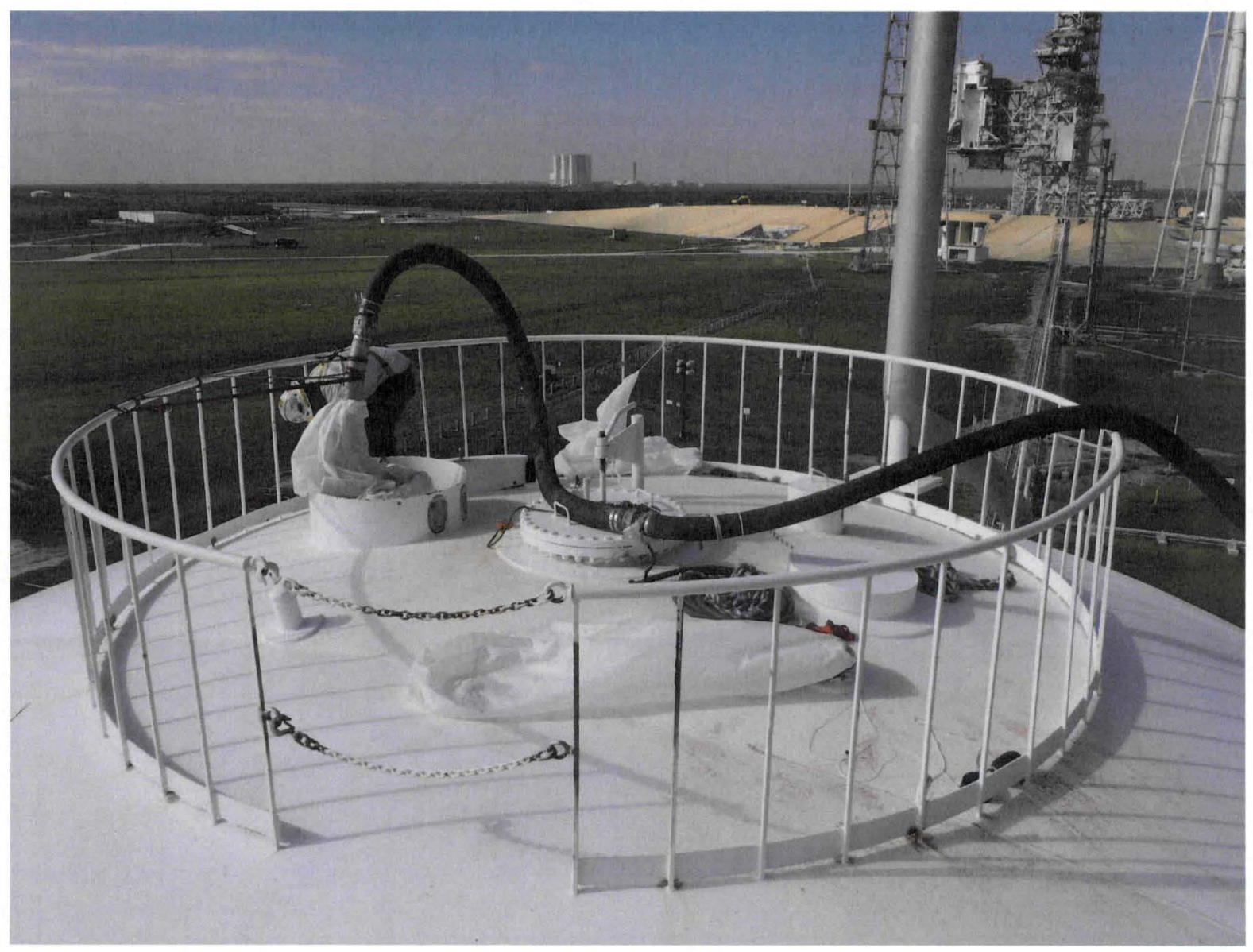

FIGURE 7. Perlite Top-off Fill and Vent Ports

\section{CONCLUSION}

After top-off was completed, an estimated 8,000 cubic feet (14\%) of perlite had been blown into the annulus. This volume was within the original estimated void range of 7,000 $-14,000$ cubic feet $(12-24 \%)$. The addition of this amount of insulation should result in a reduction in the sphere's boiloff. However, the recent cancellation of the Constellation Program means that there is no known need date for this sphere to become operational again. Until the sphere is brought back into service and experiences several months of quiescence, the exact degree to which the sphere's performance has improved will remain unknown. 